\title{
Mechanisms and solutions to the brittle solder joint in electroless Ni plating
}

\author{
Xuan Wang ${ }^{\mathrm{a}, *}$, C. Key Chung ${ }^{\mathrm{b}}$ \\ a Industrial and Manufacturing Engineering Department, California Polytechnic State University, 1 Grand Ave., San Luis Obispo 93407, USA \\ b Engineering Center, Siliconware Precision Industries Co., Ltd., No. 153, Sec 3, Chung Shan Rd., Tantzu, Taichung 42756, Taiwan
}

\section{A R T I C L E I N F O}

\section{Keywords:}

Brittle solder joint

Electroless Ni plating

Nano voids

Solder reflow

\begin{abstract}
A B S T R A C T
Brittle solder joints in Electroless Ni electroless Pd immersion Au (ENEPIG) surface finishes are one of the key reliability issues in electronics assembly. Previous characterization of the reflow process has indicated that interfacial voids formed after solder reflow are responsible for the decreases in solder joint strength. However, the mechanisms behind the formation of these voids in the ENEPIG process remain unclear. In this paper, the interaction between various aspects of the ENEPIG process and solder joint strength were investigated. Surface roughness, morphology, and nano-pitting at the interface between electroless Pd and Ni-P were characterized. The size and density of nano voids inside $\mathrm{Ni}_{2} \mathrm{SnP}$ were measured after the specimens were reflowed with $\mathrm{Sn} 4 \mathrm{Ag} 0 \cdot 5 \mathrm{Cu}$ solder ball. Additionally, high speed shear solder joint strength measurements were made. The results indicated that anion adhesion induced nano-pitting at the interface between the $\mathrm{Ni}_{2} \mathrm{SnP}$ intermetallic and Pd, resulting in the formation of a nano void layer during reflow. These interfacial voids lead to lower solder joint strength. Based on the results, a solution to prevent the brittle solder joint failures is suggested.
\end{abstract}

\section{Introduction}

Electroless Ni immersion Au (ENIG) and electroless Ni electroless Pd immersion Au (ENEPIG) surface finishes have been used extensively in high density interconnects as the surface finish for substrates or printed circuit boards. These surface finishes provide better dimensional control, surface flatness, and solder joint strength, all of which are desirable for fine pitch flip chip interconnects [1]. In comparison to electrolytic plating, electroless $\mathrm{Ni}$ (EN) plating is denser, more uniform, and has superior corrosion and wear resistance [2,3]. In passivated EN surface such as ENIG, it has been found that occasionally the brittle fracture of the solder joint can occur during thermal-mechanical testing, revealing a clean, flat $\mathrm{Ni}_{3} \mathrm{P}$ surface [4]. Analysis of the solder joint after reflow showed multiple Ni-Sn-P intermetallic compound layers at the interface between $\mathrm{Ni}_{3} \mathrm{Sn}_{4}$ and $\mathrm{Ni}-\mathrm{P}$ [5-7]. Earlier studies suggested attributed the brittle fractures to the crystallization of the Ni-P layer [8,9]. Some researchers also proposed the hyperactive corrosion-induced black pad or mud cracking on the EN surface finish as possible reasons for degraded solder joint strength [10]. More recently, researches have determined that the fracture occurs at the interface between the $\mathrm{Ni}_{2} \mathrm{SnP}$ and $\mathrm{Ni}_{3} \mathrm{P}$ layers $[4,11]$. The susceptibility to brittle fracture at this interface has been hypothesized to be caused by series of nano voids inside the $\mathrm{Ni}_{2} \mathrm{SnP}$ intermetallic compound. These voids grow and coalesce after multiple reflows and establish a continuous voiding line inside $\mathrm{Ni}_{2} \mathrm{SnP}$ [4]. Once this weakened solder joint is

\footnotetext{
* Corresponding author.

E-mail addresses: xwang12@calpoly.edu (X. Wang), keychung@spil.com.tw (C.K. Chung).
}

subsequently loaded, the characteristic brittle fracture occurs. One of the contributing factors is the inherent porosity found in the immersion Au layer that allows oxygen atoms to diffuse through to the $\mathrm{Ni}$ and cause oxidation [12]. As with ENEPIG, introducing an amorphous Pd barrier layer prevents this diffusion, effectively alleviating the Ni oxidation [13]. However, the nano voids inside the $\mathrm{Ni}_{2} \mathrm{SnP}$ intermetallic are still formed due to a hyperactive corrosion process. The purpose of this paper is to understand the origin and evolution of these nano voids. A complete characterization of the nano pitting during the surface finish process up to the solder reflow step to form the joint has been conducted. Finally, solutions to prevent the brittle solder joint failures are suggested.

\section{Experimental procedures}

The ENEPIG process consists of the following steps: Cu substrate cleaning, electroless Ni plating ( $5 \mu \mathrm{m}$ thickness), electroless Pd formation, and immersion Au deposition ( $50 \mathrm{~nm}$ thickness). The ENEPIG plating solutions used in this paper were supplied by Uyemura Corp., Japan. To provide a clean surface suitable for plating, the polished copper substrate was acid-rinsed by $10 \% \mathrm{H}_{2} \mathrm{SO}_{4}$ for 2 mins to remove any remaining oxides. The substrate was then micro-etched with a solution composed of $\mathrm{Na}_{2} \mathrm{~S}_{2} \mathrm{O}_{8} 100 \mathrm{~g} / \mathrm{L}$ and $\mathrm{H}_{2} \mathrm{SO}_{4} 40 \mathrm{~g} / \mathrm{L}$ for $1 \mathrm{~min}$ and rinsed with de-ionized (DI) water. After rinsing, the substrates were subjected to a $26{ }^{\circ} \mathrm{C}$ palladium activation solution for $3 \mathrm{~min}$. Electroless nickel in the form of a nickelphosphorus alloy was deposited onto the palladium-activated copper by immersing the substrates into an $80^{\circ} \mathrm{C}, \mathrm{pH} 4.6$ electroless nickel bath for $30 \mathrm{~min}$. Upon removal from the bath, the Ni-P specimen was rinsed in three DI water tanks sequentially. To verify the cleanliness of the 
substrate, the water conductivity of the third rinse tank was measured. To form the Pd layer, the nickel plated sample was placed in an $80^{\circ} \mathrm{C}$ electroless bath of TPD-30 (Uyemura Corp., Japan) for 15 min. After plating, the specimens were rinsed with DI water. To form the final Au layer, the Pdplated substrates were dipped into an immersion bath consisting of $1 \mathrm{~g} / \mathrm{L}$ Au with the stabilizers TSB72M (Uyemura Corp., Japan) at $100 \mathrm{~g} / \mathrm{L}$; TSB72R (Uyemura Corp., Japan) at $7 \mathrm{~mL} / \mathrm{L}$ and KCN 5 ppm. This caused a galvanic reaction to occur between the Au solution and Pd-plated surface, resulting in the deposition of the final solid Au layer. As a final step, the specimens were rinsed by DI water and air dried. The final EN layer consisted of 3-9 wt\% phosphorous and was between 5 and $9 \mu \mathrm{m}$ thick. Compositions and preparation conditions of the ENEPIG surface finishes can be found in our previous publication [13]. The surface roughness of the plated specimens was measured using Atomic Force Microscopy (AFM). The surface morphology of the Ni was analyzed with high-resolution field emission scanning electron microscopy (FESEM). The Ni-P weight percentage were measured using energydispersive X-ray spectroscopy (EDX). The plated ENEPIG was then cross-sectioned using a focus ion beam (FIB) and analyzed with a JEOL JEM-2100F transmission electron microscope (TEM). Details of a specimen's preparation for TEM analysis can be found in our earlier publication [4]. The density of the nano pitting was recorded as the number of voids per micron length. After the specimens were coated with a layer of flux, $0.5 \mathrm{~mm}$ diameter $\mathrm{Sn} 4 \mathrm{Ag} 0 \cdot 5 \mathrm{Cu}$ solder balls were placed onto the surface, followed by a $250{ }^{\circ} \mathrm{C}$ reflow for $50 \mathrm{~s}$. The interface between the solder and Ni layer was cross sectioned with a FIB and analyzed with TEM. The density of the nano voids was measured and recorded. The solder joint strength was characterized by high-speed shear (HSS) at $1500 \mathrm{~mm} / \mathrm{s}$ at zero degrees.

(a)

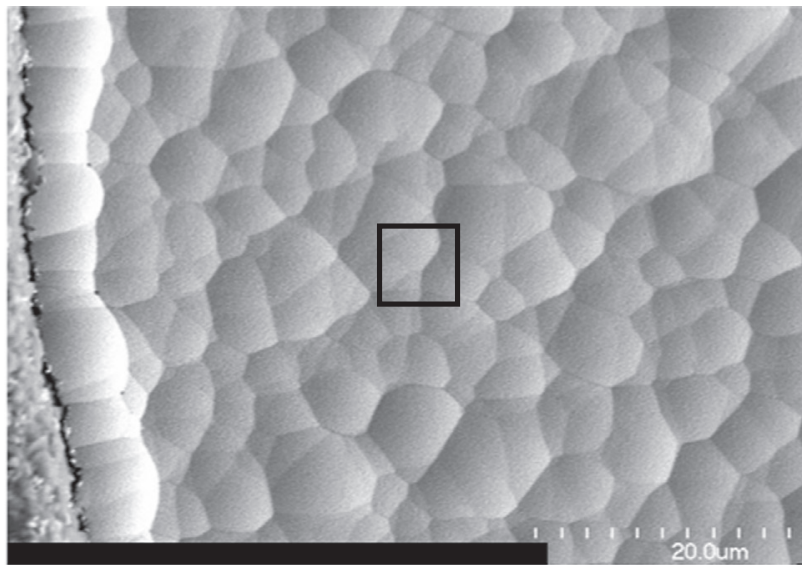

(b)

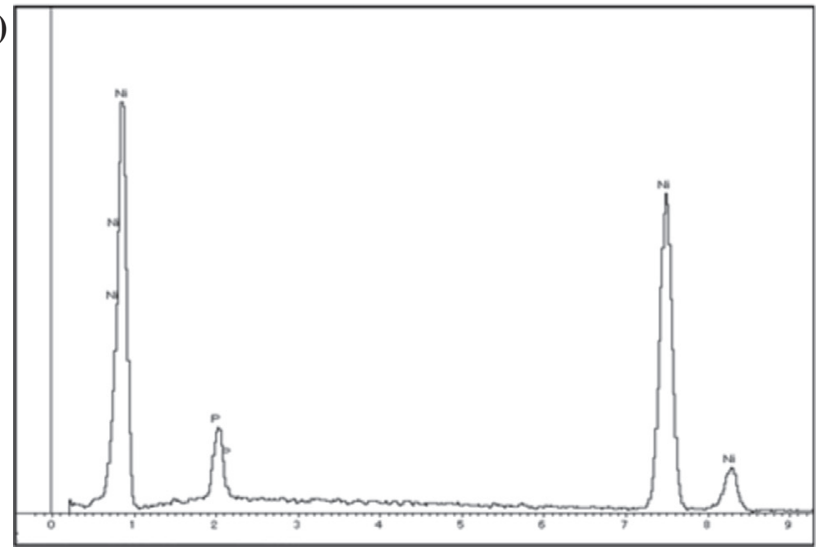

Fig. 1. SEM micrographs of (a) Ni-P nodules. (b) EDX spectrum shows $\mathrm{P}$ contents in the range of $6-9 \mathrm{wt} \%$.
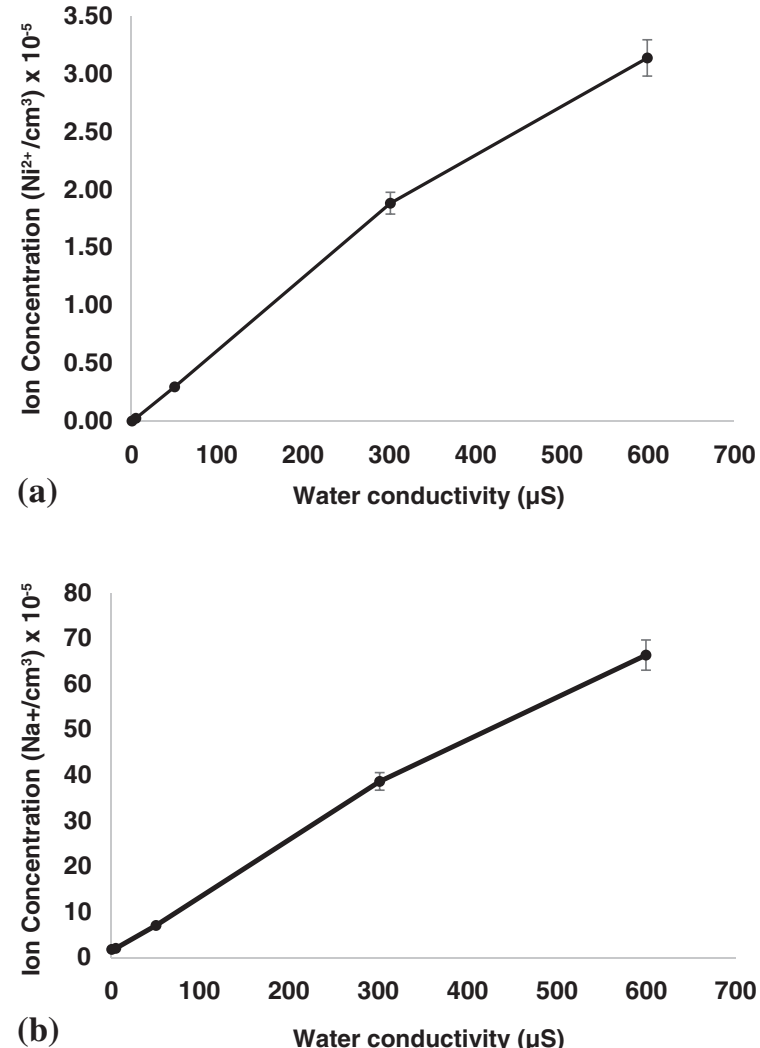

Fig. 2. Effects of ions $\left[\mathrm{Ni}^{2+}\right]$ and $\left[\mathrm{Na}^{+}\right]$concentration on water conductivity.

\section{Results}

\subsection{Characterization of electroless Ni surface}

As mentioned in the introduction, hyper corrosion of Ni can cause nano pitting. Fig. 1 is a representative FE-SEM micrograph of the nodular structure of the EN surface. EDX analysis of the $\mathrm{Ni}(\mathrm{P})$ surface returned a phosphorus composition of 6-9 wt\%. The purpose of the post Ni deposition DI water cleaning is to clean the surface of the Ni from previous plating steps and remove all residual solutions. To analyze the ion concentration in the residual solutions, the water conductivity of the final rinse tank post Ni deposition was measured. Fig. 2 shows the relationship between the water conductivity and the $\mathrm{Ni}^{2+}$ and $\mathrm{Na}^{+}$ionic concentration in the cleaning tank. The water conductivity increases as the concentration of both ions inside the DI water-rinsing bath increase. Fig. 3 shows the plot of the EN surface roughness versus water

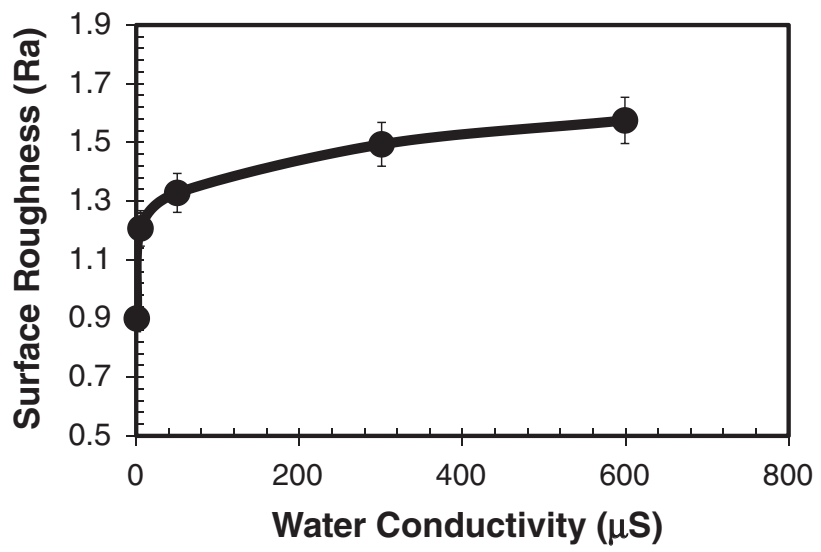

Fig. 3. Effects of water conductivity on surface roughness. 

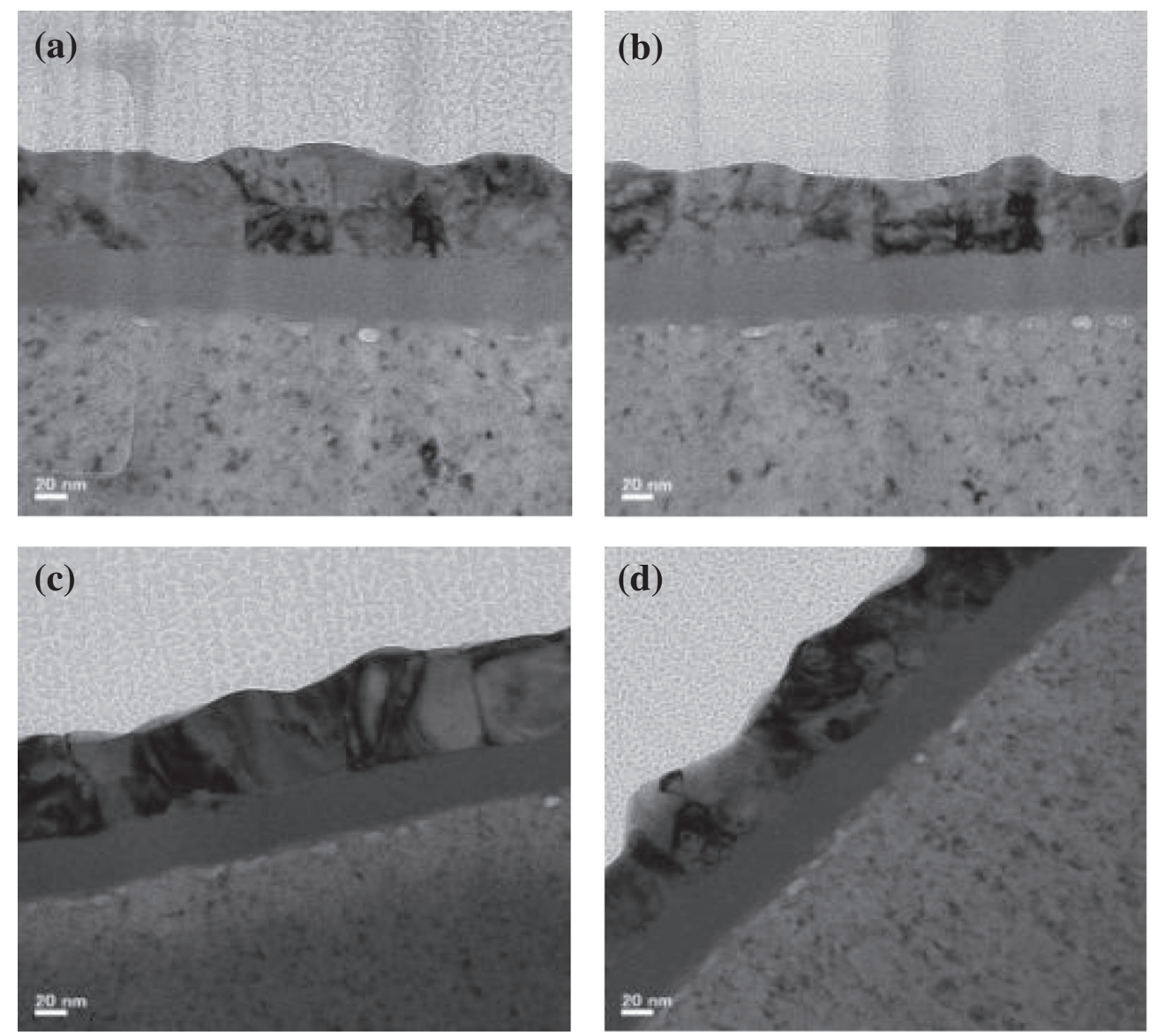

Fig. 4. TEM micrographs showing interfacial white spots at different water conductivities ( $\mu$ S): (a) 5.2; (b) 50.3; (c) 300.9 ; (d) 599.3 .

conductivity; the roughness increases exponentially with increasing water conductivity. The FIB cross sectioned samples were examined under bright-field TEM (Fig. 4a-d). White spots at the interface between the electroless Pd and the electroless Ni layers were found. Table 1 shows the measured densities of the white spots per micron length. Laurila and Vourinen [14] reported similar white spots and suggested they are voids. The sizes of the white spots are in the range of $5-$ $20 \mathrm{~nm}$ and the density of these white spots increase with both increasing surface roughness and water conductivity.

\subsection{Characterization of nano voids after reflow}

Fig. 5 is the FE-SEM depicting a micrograph of the solder joint after reflow. The micrograph shows connected interfacial nano voids inside the $\mathrm{Ni}_{2} \mathrm{SnP}$ layer. Fig. $6 \mathrm{a}-\mathrm{d}$ show the high resolution images of various FE-SEM micrographs; Table 2 lists the density of the nano voids based on analysis of the micrographs. The size of the nano voids is from 4 to $20 \mathrm{~nm}$. As the water conductivity increases, the nano void density increases as well. The sizes nano voids are very similar to the size of the

Table 1

The measured density of interfacial voids and water conductivity before solder reflow.

\begin{tabular}{|c|c|c|c|}
\hline $\begin{array}{l}\text { TEM } \\
\text { micrographs }\end{array}$ & $\begin{array}{l}\text { Water } \\
\text { conductivity } \\
(\mu S)\end{array}$ & $\begin{array}{l}\text { Size of interfacial } \\
\text { white spot }(\mathrm{nm})\end{array}$ & $\begin{array}{l}\text { Density of interfacial white } \\
\text { spot (spots } / \mu \mathrm{m})\end{array}$ \\
\hline (a) & 5.2 & $8-14$ & 19 \\
\hline (b) & 50.3 & 5-17 & 30 \\
\hline (c) & 300.9 & $6-16$ & 44 \\
\hline (d) & 599.3 & $8-20$ & 52 \\
\hline
\end{tabular}

white spots at the interface between electroless Pd and Ni-P before reflow as depicted in Fig. 4.

However, the density of the nano voids per micron after reflow is lower than the density before reflow. On the specimen with the lowest water conductivity, no interfacial nano voids were observed. Fig. 7 shows the HSS energy across different interfacial nano void densities; as the interfacial nano void density increases, the energy drops significantly. At the density of about 9 nano voids per micron, further increases in the interfacial nano void density does not result in further degradation of the HSS energy. Above this nano void density, the solder joints exhibit brittle fracture failures.

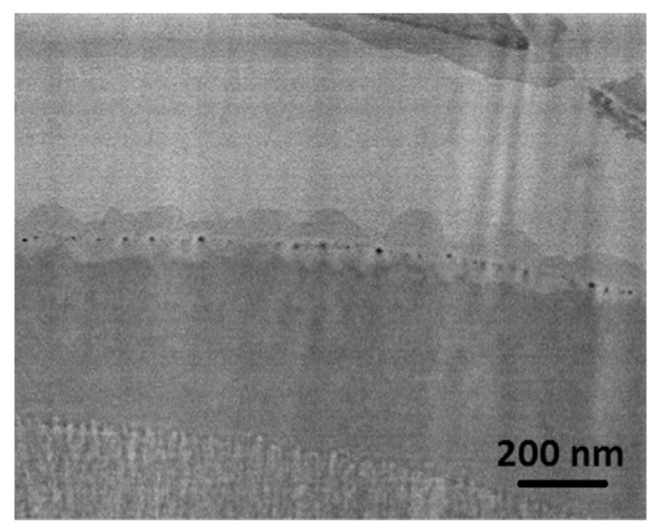

Fig. 5. High resolution image of a FE-SEM micrograph depicting interfacial nano voids at the $\mathrm{Ni}_{2} \mathrm{SnP}$ after reflow. 

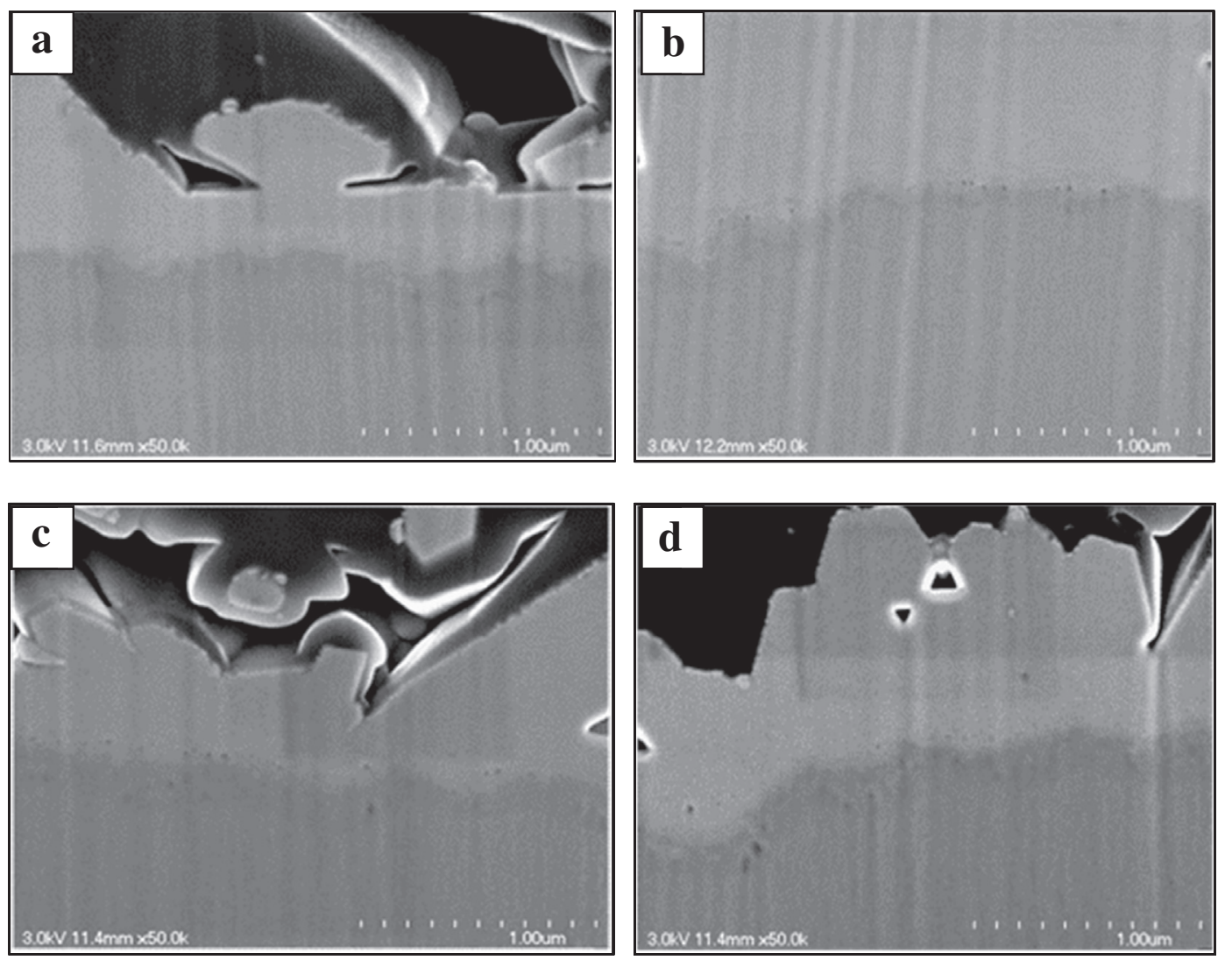

Fig. 6. High resolution images of FE-SEM micrographs showing interfacial voids found inside the $\mathrm{Ni}_{2} \mathrm{SnP}$ at different water conductivities ( $\mu$ S): (a) 5.2 ; (b) 50.3 ; (c) 300.9 ; (d) 599.3 .

\section{Discussions}

\subsection{Effects of residual ion concentration on nano pitting}

Fig. $2 \mathrm{a}$ and $\mathrm{b}$ show that as the $\mathrm{Ni}^{2+}$ ion $/ \mathrm{cm}^{-3}$ concentration increases, the water conductivity of the third rinse tank after EN increases in accordance with standard ohmic law [15]. The $\mathrm{Na}^{+}$ions are possibly from the previous EN plating bath; the presence of $\mathrm{Ni}^{2+}$ suggests that the $\mathrm{Ni}^{2+}$ originates from the dissolution of $\mathrm{Ni}$ due to incomplete residual EN plating solution removal prior to the electroless Pd plating step. As a result, the dissolution of $\mathrm{Ni}$ and the residual plating solution in the DI water rinse manifests as an increase in water conductivity. The EN surface roughness shown in Fig. 3 also supports this hypothesis. Therefore, the corrosion of $\mathrm{Ni}$ is likely to happen during the water rinsing process. The poorer corrosion performance of EN specimens prepared can be attributed to the presence of the particle boundaries and the trace impurities adsorbed onto or included inside the forming the Ni-P layer [4]. Schlaup and Horch [16] analyzed the phosphate adsorption in-situ using scanning transmission microscopy. The size of the anions observed as white small patches was similar to our findings: approximately $5 \mathrm{~nm}$. TEM images such as those depicted in Fig. 4 shows the white spots that were inferred to be nano pitting. During

Table 2

The measured density of interfacial voids and water conductivity after solder reflow.

\begin{tabular}{llll}
\hline $\begin{array}{l}\text { TEM } \\
\text { micrographs }\end{array}$ & $\begin{array}{l}\text { Water conductivity } \\
(\mu \mathrm{S})\end{array}$ & $\begin{array}{l}\text { Nano pitting size } \\
(\mathrm{nm})\end{array}$ & $\begin{array}{l}\text { Pitting density } \\
(\text { spots/ } \mu \mathrm{m})\end{array}$ \\
\hline (a) & 5.2 & 0 & 0 \\
$(\mathrm{~b})$ & 50.3 & $5-17$ & 4.6 \\
$(\mathrm{c})$ & 300.9 & $6-16$ & 5.2 \\
$(\mathrm{~d})$ & 599.3 & $8-20$ & 6.0 \\
\hline
\end{tabular}

the electroless Pd process, the Pd layer deposits on top of the nano pitting, which trapped the impurities and anions.

\subsection{Evolution of nano pitting during reflow}

During reflow, the dissolution of Au and Pd helps to close some of the voids. This can be observed by comparing the nano pitting before reflow and after reflow as shown in Tables 1 and 2. The nano pitting produced voids that were trapped at the interface within the $\mathrm{Ni}_{2} \mathrm{SnP}$ layer, as studied by Kang et al. [17]. Our study found that flux can further mitigate nano pitting, albeit not entirely. This may be because the immersion $\mathrm{Au}$ and electroless Pd cover the top surface of the nano pitting, acting as an effective barrier of which the flux can clean [13]. As seen in Fig. 6, the size of the nano white spots is very similar to the interfacial nano voids after reflow. The number of nano voids per unit micron was

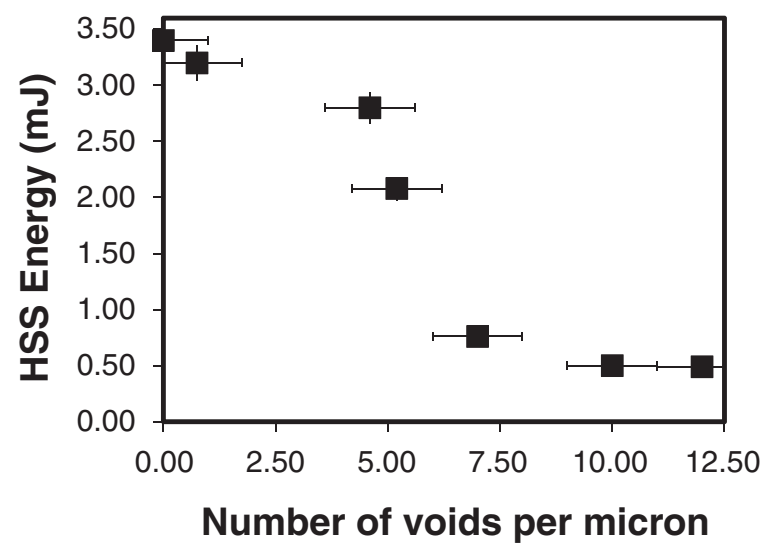

Fig. 7. Plot of solder joint strength (HSS energy) at different nano void densities. 
reduced after reflow. One method to reduce the water conductivity is to increase the amount water rinsing. Thus, the purity, temperature, and air agitation are critical factors for the DI water used to remove the adhered anions. Fig. 7 shows that as the void density increases, the HSS energy decreases accordingly because of the decreasing effective area connecting the layers to the substrate [4]. It was determined that the presence of 9-12 nano voids was sufficient for the specimens to fail the shock tests.

\section{Conclusions}

The interfacial nano voids at the intermetallic $\mathrm{Ni}_{2} \mathrm{SnP}$ layer was confirmed to originate from existing nano pitting. The cause of the nano pitting was attributed to anion adhesion that resulted in Ni dissolution and subsequent rough surface formation. The level of Ni dissolution can be monitored analyzing the post Ni deposition cleaning water conductivity. The higher the water conductivity in the DI water, the rougher the Ni-P surface produced. During soldering, the nano pits became trapped at the $\mathrm{Ni}_{2} \mathrm{SnP}$ interface, forming a layer of voids and induced brittle solder joint failures. It was found that a higher nano void density is correlated to a higher water conductivity. Higher nano void density caused brittle solder failures joint due to the reduction of the effective connected area in the solder joint. By enhancing the water rinsing process after the Ni-P plating step, the anion adhesion prior to the Immersion Au plating can be alleviated. As a result, the pitting effect and the nano void density inside the $\mathrm{Ni}_{2} \mathrm{SnP}$ layer can be mitigated. Thus, it is important to monitor the water conductivity of the rinse after the Ni-P plating step and prior to electroless Pd step in the ENEPIG process.

\section{Acknowledgements}

The authors would like to thank Mr. David Otsu from California Polytechnic State University San Luis Obispo for his proof-reading of the manuscript.

\section{References}

[1] G.O. Mallory, The fundamental aspects of electroless nickel plating, in: G.O. Mallory, J.B. Hajdu (Eds.), Electroless Plating: Fundamental and Application, Noyes Publications/William Andrew Publishing LLC, New York 1991, pp. 1-56.

[2] R. George, S. Venkatachalam, K.N. Ninan, Electrochemical impedance measurements on Ni-P coated magnesium alloy, chromated magnesium alloy, and anodised aluminium alloys in aqueous salt solutions, Br. Corros. J. 37.1 (2002) 37-42.

[3] W.-J. Cheong, B.L. Luan, D.W. Shoesmith, Protective coating on Mg AZ91D alloy-the effect of electroless nickel (EN) bath stabilizers on corrosion behavior of Ni-P deposit, Corros. Sci. 49 (2007) 1777-1798.

[4] C.K. Chung, Y.J. Chen, W.M. Chen, C.R. Kao, Origin and evolution of voids in electroless Ni during soldering reaction, Acta Mater. 60 (2012) 4586-4593.

[5] P.L. Liu, Z. Xu, J.K. Shang, Thermal stability of electroless-nickel/solder interface part a: interfacial chemistry and microstructure, Metall. Mater. Trans. 31A (2000) 2857-2866.

[6] Y.-C. Lin, J.-G. Duh, Phase transformation of the phosphorus-rich layer in $\mathrm{SnAgCu} / \mathrm{Ni}$ P solder joints, Scr. Mater. 54 (2006) 1661-1665.

[7] C.W. Hwang, K. Suganuma, Interface microstructures between Ni-P alloy plating and Sn-Ag-(Cu) lead-free solders, J. Mater. Res. 18 (2003) 2540-2543.

[8] J.W. Jang, P.G. Kim, K.N. Tu, D.R. Frear, P. Thompson, Solder reaction-assisted crystallization of electroless Ni-P under bump metallization in low cost flip chip technology, J. Appl. Phys. 85 (1999) 8456-8463.

[9] K.C. Hung, Y.C. Chan, C.W. Tang, H.C. Ong, Correlation between Ni3Sn4 intermetallics and Ni3P due to solder reaction-assisted crystallization of electroless Ni-P metallization in advanced packages, J. Mater. Res. 15 (2000) 2534-2539.

[10] K. Zeng, R. Stierman, D. Abbott, M. Murtuza, The root cause of black pad failure of solder joints with electroless Ni/immersion gold plating, JOM 58 (2006) 75-79.

[11] H.-B. Kang, J.-H. Bae, J.-W. Yoon, S.-B. Jung, J. Park, C.-W. Yang, Characterization of ternary Ni2SnP layer in Sn-3.5Ag-0.7Cu/electroless Ni (P) solder joint, Scr. Mater. 63 (11) (2010) 1108-1111.

[12] R. Ramanauskas, A. Selskis, J. Juodkazyte, V. Jasulaitiene, PCB failure analysis related to the ENIG black pad problem, Circuit World 39 (3) (2013) 124-132.

[13] C.C. Li, W.L. Shih, C.K. Chung, C.R. Kao, Amorphous Pd layer as a highly effective oxidation barrier for surface finish of electronic terminals, Corros. Sci. 83 (2014) 419-422.

[14] T. Laurila, V. Vuorinen, Combined thermodynamic-kinetic analysis of the interfacial reactions between Ni metallization and various lead-free solders, Materials 2.4 (2009) 1796-1834.

[15] R.L. Miller, W.L Bradford, N.E. Peters, Specific conductance: theory considerations and application to analytical quality control, US Department of Interior, Geol. Surv. Water Supply Pap. 2311 (1988) 1-16.

[16] C. Schlaup, S. Horch, In-situ STM study of phosphate adsorption on $\mathrm{Cu}(111)$, $\mathrm{Au}(111)$ and $\mathrm{Cu} / \mathrm{Au}(111)$ electrodes, Surf. Sci. 608 (2013) 44-54

[17] H.-B. Kang, J.-H. Bae, J.-W. Lee, M.-H. Park, J.-W. Yoon, S.-B. Jung, C.-W. Yang, Characterization of interfacial reaction layers formed between $\mathrm{Sn}-3.5 \mathrm{Ag}$ solder and electroless Ni-immersion Au-plated Cu substrates, J. Electron. Mater. 37 (1) (2008) 84-89. 